\title{
AUTOMATIC SUMMARIZATION OF KOREAN NEWS ARTICLES USING EXTRACTIVE- ABSTRACTIVE BINDING MODEL
}

\author{
Meiying Ren ${ }^{1}$ and Sinjae $\mathrm{Kang}^{2 *}$ \\ ${ }^{1}$ Department of Computer \& Information Engineering, Daegu University \\ ${ }^{2}$ Department of Computer \& Information Engineering, Daegu University \\ *sjkang@daegu.ac.kr
}

\begin{abstract}
The approach for text summarization can be broadly divided into extractive and abstractive summarization. The extractive method selects summary sentences from the original text, and the abstractive method generates new summary sentences through learned models. Extractive summarization is easy to implement, but the generated summary is unnatural, while the abstractive method generates natural summaries but is difficult to implement. The encoder-decoder model, which is the most commonly used abstractive method, has a disadvantage in that performance is degraded when the input is too long. Therefore, in this paper, we propose a method of extracting important sentences from the input document through the extractive model first, and then performing abstractive summarization by inputting the generated extraction summary. The extractive model is based on the TextRank graph method, and the abstractive summarization system is based on the recurrent neural network (RNN) Encoder-Decoder model. We applied the abstractive model and the extractive-abstractive binding model to Korean newspaper articles. We used 15,000 online news articles as the dataset. 13,500 articles were used for training, and 1,500 were used for testing. News highlights written by human reporters are considered gold summaries. Experimental results show that the proposed system provides better results than the abstractive summarization only.
\end{abstract}

Keywords - Text Summarization, RNN Encoder-Decoder, LSTM, TextRank, Binding Model

\section{INTRODUCTION}

Automatic document summarization is the process of extracting concise, natural summaries from long documents. There are two main approaches to automatic text summarization: extractive and abstractive. The extractive method extracts the main sentences from the original text. Since the extraction unit is the sentence, the sentence itself is natural, but the seams and flows among sentences may be awkward. By contrast, the abstractive summary learns information from the original text and generates new summary sentences through language modeling.

Abstractive summarization is much more complicated than ex-tractive summarization, starting with the model design; but in recent years, many studies have dealt with abstractive summarization in order to overcome the unnaturalness and generate more concise summaries, compared to extracted summaries. To supplement the shortcomings

Received: January 8, 2019

Reviewed: March 4, 2019

Accepted: March 12, 2019

* Corresponding Author 
of linguistic knowledge-based methods, which are costly in terms of both time and money, machine learning techniques are mainly used. In particular, an abstractive model using the recurrent neural network (RNN) is emerging as a trend, along with recent developments in deep learning $[1,2,3]$.

Despite expectations that automatic summarization of a document would have greatly improved along with the development of deep learning, the text summarization task is not easy. It should be based on a huge amount of information, while not stepping outside the input document information boundary. A simple model of the Input Layer-Long ShortTerm Memory (LSTM) Layer-Output Layer structure does not perform well. The difficulty is mainly because a sufficient amount of information cannot be compressed in the content vector of a limited dimension when the input is long. The technique of using the output together with the content vector proposed in [1] and [4] showed quite good results. Since then, IBM [2] and Google [3] have obtained better results by adding to the model hierarchical attention and a pointing mechanism.

In this paper, we propose an automatic summarization model that combines the extractive method and the abstractive method in order to compensate for the difficulty of the abstractive model that occurs as the input becomes longer, as presented in previous studies. First, important sentences are extracted from a long article through extractive summarization. Next, abstractive summarization is performed by inputting the summary com-posed by the extractive system. The outcome of the extractive model reduces the burden on the abstractive process by reducing the range of input with important sentences. The extraction summary uses the TextRank [5] graph model, which is widely used and known for its fine performance, and the abstractive system applies the model in [1] mentioned above. The task does not generate a title from the first $\mathrm{n}$ sentences, which was done in the existing papers [2,3], but invokes a process of inputting the entire article and generating a summary that is much longer than the title. Therefore, the problem definition is different, and the latter is more difficult.

\section{LITERATURE REVIEW}

\subsection{AUTOMATIC EXTRACTIVE TEXT SUMMARIZATION}

Extractive automatic summarization is a matter of sentence se-lection in brief. To determine the importance of sentences, they are scored and ranked, and a certain percentage of the sentences are selected. The key to this method is the process of assigning scores to each sentence, and papers using various qualities (such as word frequency, document frequency, and sentence location) were presented before. The most commonly used technique is the TextRank [5] algorithm, which applies a graph-based PageRank [6] algorithm to text summarization. In TextRank, the node of the graph is each sentence, and the weights are mainly determined by word frequency and document frequency. Many modified TextRank algorithms have been pro-posed [7, 8], and Figure 1 is an example of a TextRank graph. The boldface numbers represent each sentence. Based on the similarity between the sentences, the text rank value of each sentence (in square brackets in Figure 1) is calculated with Equation 1:

$$
T R\left(V_{j}\right)=(1-d)+d * \sum_{V_{j} \in m_{i}\left(V_{j}\right)} \frac{w_{j i}}{\sum_{V_{k} \in \cos \left(V_{j}\right)} w_{f k}} T R\left(V_{j}\right)
$$




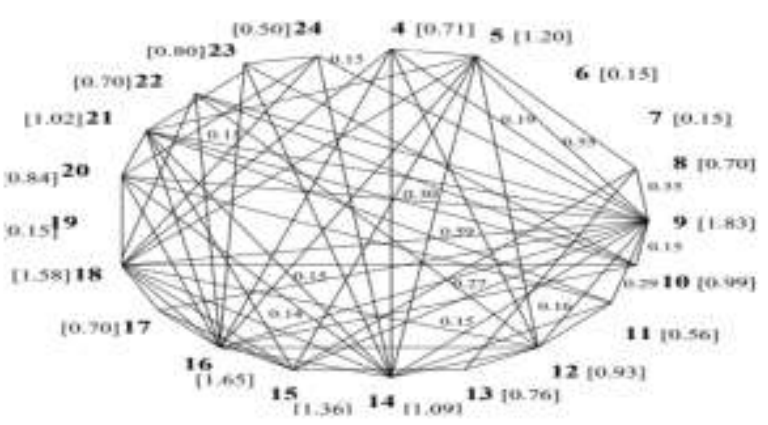

Fig. 1 TextRank Graph Sample [5].

Similarity-based graph methods are often used in extractive summarization and show fine results. However, since a sentence itself cannot be modified, there is a problem with unnaturalness (or missing information).

\subsection{ABSTRACTIVE TEXT SUMMARIZATION}

To overcome the drawback of an unnatural summary generated by the extractive model, the abstractive technique is used. There are linguistic and statistical methods in the abstractive process. Linguistic techniques require a large knowledge base built by experts, which is costly and time consuming. Therefore, most studies use statistical techniques, i.e. machine learning techniques. Machine learning techniques, such as Naïve Bayes [9] and the Markov model [10], have been used, but still have to be based on a large knowledge base, and there is an enormous amount of computation required in language modeling. Thus, recent studies use a neural network to reduce the dimension.

2.2.1. RECURRENT NEURAL NETWORK (RNN): There are two types of neural network that are widely used. One is the convolutional neural network $(\mathrm{CNN})$, which performs convolution and pooling to reduce the size of the input layer; the other is the RNN, which accepts the output generated in the previous step as input in the subsequent step. The CNN is mainly applied to classification problems and is used when the output of the previous stage is independent from the output of the latter stage [11, 12]. To conduct automatic summarization, language generation is necessary. The generated word greatly influences which word to generate next in all kinds of languages. In other words, because natural language data can be viewed as time series data, the RNN [13] is mainly used for language generation. An RNN uses the result generated in the previous step as input for the next step, as shown in Fig. 2.
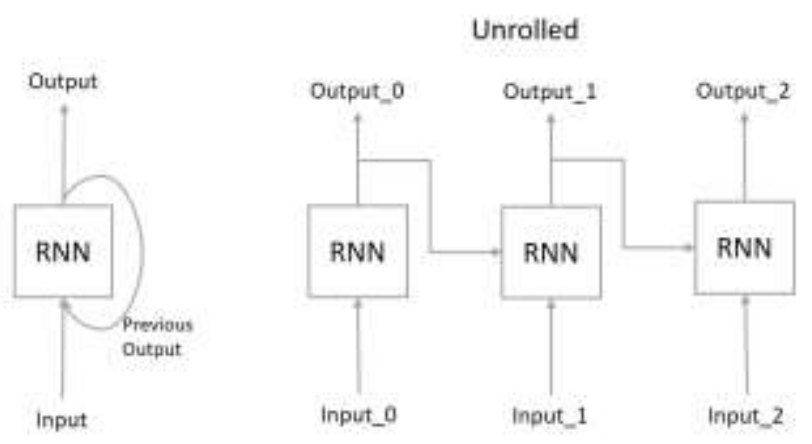

Fig. 2: Recurrent Neural Network [13] 
The most commonly used RNN cell is Long Short-Term Memory (LSTM) [14]. LSTM was proposed by Hochreiter and Schmidhuber [15] in 1997 and developed by Gers et al. [14]. In recent years, the performance has been proven as it has been used in large global IT companies such as Google [16] and Apple [17] for machine translation, personal assistant robots, and so on. LSTM consists of a memory cell, an input gate, an output gate, an update gate, and a forgetting gate. The internal gates determine whether to store the input in memory or be forgotten, or what rate of input to store, etc. LSTM has solved the problem of gradient vanishing, to some extent, because it can store old data in a memory cell.

2.2.2 RNN ENCODER-DECODER MODEL: Unlike machine translation, abstractive summarization (based on sufficiently piled up human knowledge and which should remain within the input document's information boundary) is not an easy task. A simple input-LSTM-output model of the output structure does not provide much performance. However, machine translation and document summary are basically similar because basic problem definition in both tasks is sequence-to-sequence generation. Therefore, we used RNN Encoder-Decoder [4, 18], which has shown good performance in ma-chine translation in English, to perform automatic summarization. The encoderdecoder model encodes an input document into a context vector of a certain length through an encoder, and then generates a new word by inputting the context vector and previously generated word into a decoder. The difference from machine translation is that the input and output predict the vocabulary in two different languages, and machine translation needs to find the entire vocabulary of the language, while the automatic summarization should not deviate from or distort the contents of the input document.

Figure 3 shows the RNN Encoder-Decoder abstractive summarization model [1, 2]. The left part of Context Vector is the encoder, and the right part is the decoder. The very first decoder input is usually a random initialized vector or a start tag vector.

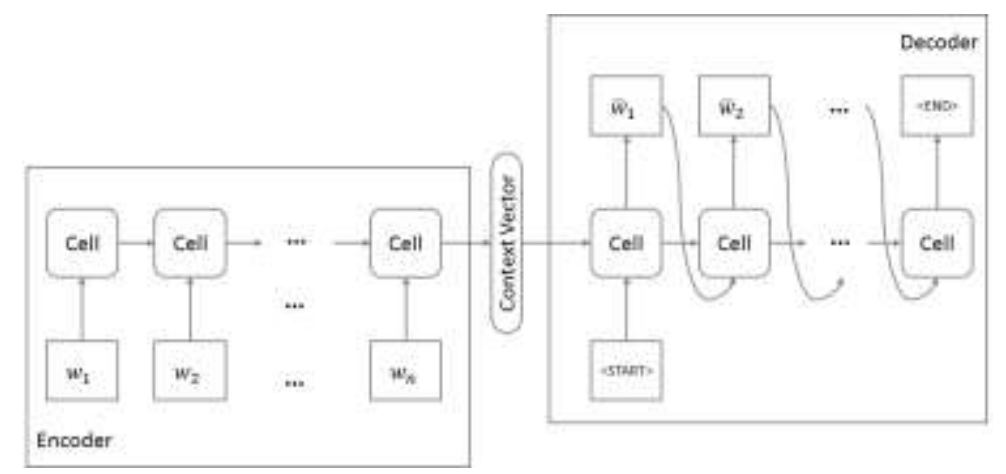

Fig. 3 RNN Encoder-Decoder Text Summarization Model

2.2.3. ABSTRACTIVE SUMMARIZATION SYSTEM USING RNN ENCODERDECODER: The encoder-decoder model [4], which was first applied in ma-chine translation, has been applied consistently to the summarization problem, obtaining notable results [1, 2]. Rush et al., [1] applied machine translation model [4] to the long sentence summarization, and Nallapati et al., [2] adopted it to the document summarization using DUC-2004 data and Gigaword data. The data were newspaper article data, and the authors viewed the input as the first $\mathrm{n}$ sentences of the news body, considering the headline of the news as a summary. Some authors constructed a new model by adding hierarchical attention [2] and a pointing algorithm [3] to existing models $[1,4]$. The Attention layer is a network that learns the importance of inputs separately, assuming there is a difference in the importance of input. The pointing algorithm calculates the probability of generating words from the vocabulary, and then combines it 
with the attention distribution to obtain the final distribution for final prediction. In this process, the learned network points to the word position in the fixed vocabulary. Figure 4 shows the Attention algorithm added to the encoder-decoder model.

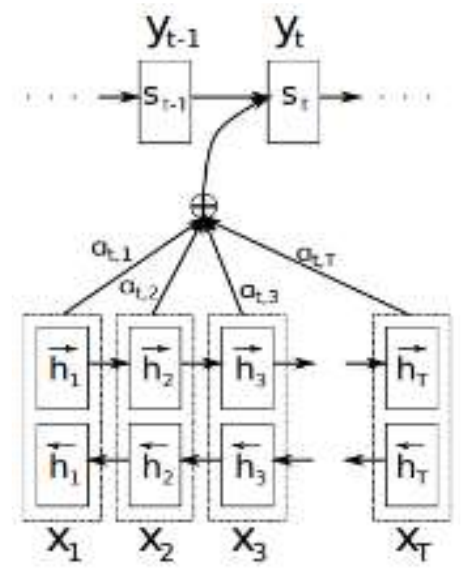

Fig. 4 RNN Encoder-Decoder Text Summarization Model with Attention [4]

\subsection{ROUGE EVALUATION}

Recall-Oriented Understudy for Gisting Evaluation (ROUGE) is often used to evaluate text summarization [19]. ROUGE has many variants. In this paper, we used ROUGE-N, the number of overlapping $\mathrm{N}$-grams $(\mathrm{N}=\{1,2, \ldots\})$ between the system sum-mary and the actual summary, and ROUGE-L, the number of overlapping Longest Common Subsequence (LCS).

\section{ROUGE-N:}

$$
\frac{\sum_{S \in\{\text { References }\}} \sum_{\text {gram }_{n}} \text { Count }_{\text {mateh }}\left(\text { gram }_{n}\right)}{\sum_{S \in\{\text { References }\}} \sum_{\text {gram }_{n}} \text { Count }\left(\text { gram }_{n}\right)}
$$

Countmatch(gramn) returns 1 if gramn, one of the n-grams in corrective summary References, is included in the n-gram set of the system summary; otherwise, it returns zero. Similarly, ROUGE-L is given by equations (3), (4), and (5).

\section{Rouge-L:}

$$
\begin{aligned}
& R_{\text {ICs }}=\frac{L C S\left(\text { Re } \int e r e n c e, S y s t e m\right)}{m} \\
& P_{\text {tos }}=\frac{\text { I.CS(Roprerpene, Systrm) }}{n} \\
& F_{l c s}=\frac{2 R_{l c s} P_{l c s}}{R_{l c s} P_{l c s}}
\end{aligned}
$$

Reference stands for the gold summary set, and System stands for the system summary. LCS(Reference, System) refers to the length of the longest common subsequence of Reference and System; $m$ is the length of the gold summary, and $n$ is the length of the system summary. 


\section{AUTOMATIC NEWS SUMMARIZATION SYSTEM USING EXTRACTIVE-ABSTRACTIVE BINDING MODEL}

\subsection{EXTRACTIVE-ABSTRACTIVE AUTOMATIC SUMMARIZATION MODEL}

The problem in the RNN Encoder-Decoder model is the out-of-vocabulary (OOV) problem, in which words that have not been shown (or low-frequency words) are not sufficiently learned. Another main problem is to overcome the phenomenon where the model cannot be learned properly if the input is long. This causes information loss, because all documents with different input lengths are encoded with the same length of content vector. In this paper, we try to summarize the model by inputting the whole article instead of the first $\mathrm{n}$ sentences $[2,3]$. We also try to generate a longer summary, rather than a headline.

The proposed automatic summary model consists of two parts. The first is the extractive summarization. In order to prevent the loss of major information, the summarization rate for extractive summarization is increased to $50 \%$, whereas the usual rate is $30 \%$. This part is implemented using the TextRank algorithm provided by the Gensim library. In the abstractive portion, we use the summary generated by the extractive model.

The subsequent abstractive summarization uses the model pro-posed in [1]. The words with low frequency are replaced with the <unk> tag (unknown) to off-load the burden of the output layer by reducing its dimension, while maintaining the maxi-mum input information to be encoded by the extraction summary.

The structure is shown in Figure 5. The abstractive part has two kinds of inputs. $<$ Summarized source doc $>$ is the extractive summaries created by the extractive part, and $<$ Summary Doc > is gold standard summaries, which are created by human reporters. Two embedding layers of the abstractive model are different because the input vocabulary and target vocabulary differ in word type and size. The dotted line means loading the hidden state of LSTM1 into LSTM2. In the experiment, only the abstractive part was used as the baseline. The word is used as the language feature, and the LSTM hidden unit size is 128 . Batch size is 64 , and epoch is 50 for both models.

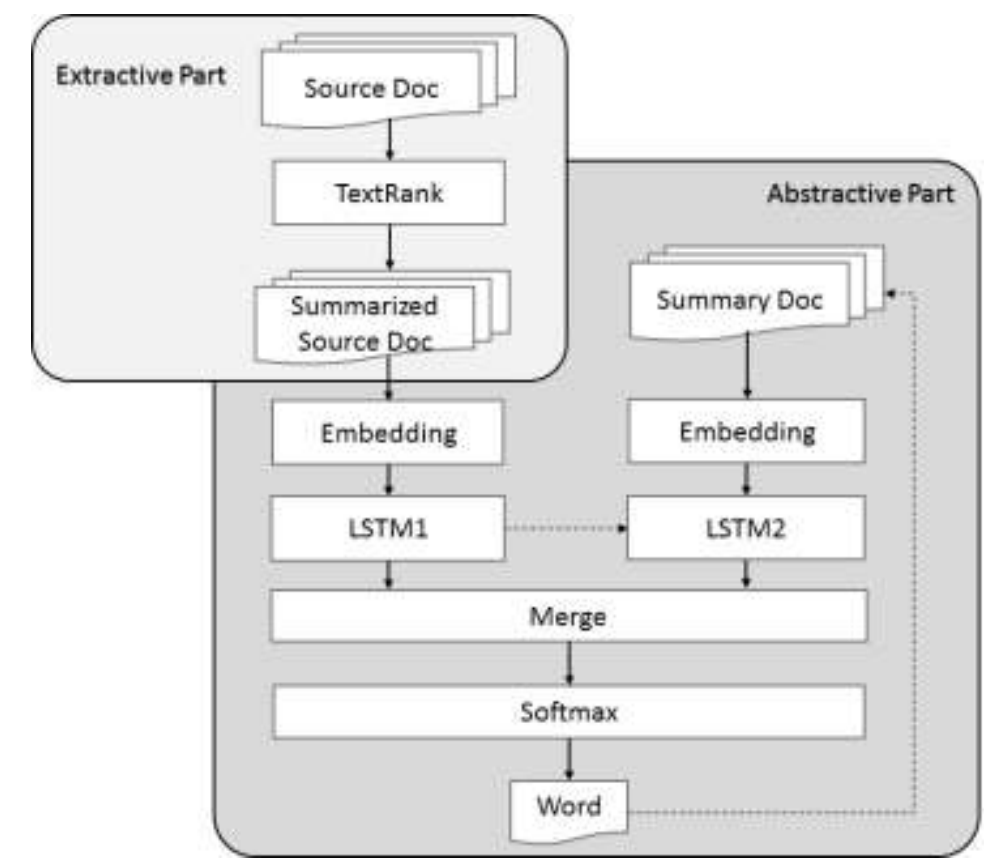

Fig. 5 Extractive-Abstractive Binding Summarization Model 


\subsection{EXPERIMENT ENVIRONMENT}

We used 15,000 Korean news consisting of political and social fields to test the proposed model. The input was the body of the news, and the output was the highlights written by the reporter. The input document vocabulary used the 5,000 most frequent words and the summary vocabulary used the top 2,000. The ratio of learning-to-test data was 9:1.

The Gensim TextRank Summarizer was used for extractive summarization, and Keras was used for the abstractive system. Tensorflow was used as the backend of Keras. The GP-GPU used in the experiments was the Nvidia GTX 1080.

Table I. Data Description

\begin{tabular}{c|c|c|c|c}
\hline & \multicolumn{2}{|c|}{ Train } & \multicolumn{2}{c}{ Test } \\
\hline Type & Sentence & Word & Sentence & Word \\
\hline Politics news dataset & 59,848 & $1,618,056$ & 7,311 & 201,741 \\
Society news dataset & 217,663 & $4,235,936$ & 21,360 & 430,572 \\
\hline
\end{tabular}

\subsection{RESULTS AND DISCUSSION}

The experimental results are shown in Table 2. In the proposed model, ROUGE-2 results showed a $4.15 \%$ improvement and ROUGE-L performance was $7.84 \%$ better than the baseline on politics news. On society news, ROUGE-2 showed a $1.92 \%$ and ROUGEL was $7.01 \%$ improvement.

This shows that extractive summarization effectively extracts important sentences, and that not much information was lost. It also explains how the length of the input has a relatively large impact on the performance of abstractive summarization.

In other words, it seems that the summary generated by the extractive system provided a sufficient amount of important in-formation necessary for abstractive summarization, while reducing the amount of input data compared to the simple abstractive summary system.

Therefore, based on the experiments, it became possible to obtain better results using abstractive summarization to solve the unnaturalness of the extractive model, i.e., reducing the calculation and memory burden of the abstractive system by using extractive summarization. Memory burden refers to the problem stated before, which is memorizing old data due to the long input.

Table II. Rouge Results of Korean News Article Summarization

\begin{tabular}{c|c|c|c}
\hline \multicolumn{2}{l|}{} & $\begin{array}{c}\text { Baseline } \\
\text { (Only Abstractive) }\end{array}$ & $\begin{array}{c}\text { Extractive- } \\
\text { Abstractive System }\end{array}$ \\
\hline $\begin{array}{c}\text { Politics news } \\
\text { dataset }\end{array}$ & Rouge-2 & 2.46 & 6.61 \\
\hline Society news & Rouge-L & 10.20 & 18.04 \\
\hline dataset & Rouge-2 & 4.36 & 6.28 \\
\hline
\end{tabular}

\section{CONCLUSION}

In this paper, we constructed a summary system that combines extractive and abstractive methods and applied it to Korean news summarization. In order to solve the problem of poor performance when input for the RNN Encoder-Decoder automatic summary model is long, the extractive summarization is performed before the abstractive model summarizes the document. As a result, the proposed system combining the two systems showed better results. ROUGE-L performance was improved by an average of $7.4 \%$, and ROUGE- 2 was $3.0 \%$. This shows that the extractive summary effectively compresses the input information for the abstractive summary. 
Future research will explore optimal vocabulary sizes for automatic Korean summaries, and will apply techniques known to help improve performance in abstractive summaries, such as the Attention layer.

\section{ACKNOWLEDGEMENT}

This research was supported by Basic Science Research Pro-gram through the National Research Foundation of Korea (NRF) funded by the Ministry of Education (NRF2016R1D1A1B03930346).

\section{REFERENCES}

[1] A. M. Rush, S. Chopra and J. Weston, "A Neural Attention Model for Abstractive Sentence Summarization", Proceedings of the 2015 Conference on Empirical Methods in Natural Language Processing, (2015), $\quad$ pp. 379-389, available http://www.emnlp2015.org/proceedings/EMNLP/pdf/EMNLP044.pdf, last visit:21.12.2018

[2] R. Nallapati, B. Zhou, C. Santos, C. Gulcehre, and B. Xian, "Ab-stractive Text Summarization Using Sequence-To-Sequence RNNs and Beyond", Proceedings of the 20th SIGNLL Conference on Computational Natural Language Processing(CoNLL), (2016), pp. 280-290, available online: http://www.aclweb.org/anthology/K16-1028, last visit:21.12.2018

[3] A. See, P. J. Liu, and C. D. Manning, "Get to The Point: Summari-zation with Pointer-Generator Networks", Proceedings of the 55th Annual Meeting of the Association for Computational Linguistics, Vol. 1: Long Papers, (2017), pp.1073-1083, http://dx.doi.org/10.18653/v1/P17-1099

[4] D. Bahdanau, K. Cho, and Y. Bengio, "Neural Machine Translation by Jointly Learning to Align and Translate", ICLR 2015: Interna-tional Conference on Learning Representations 2015, (2015), pp. 1-15, available online: https://arxiv.org/pdf/1409.0473.pdf, last visit: 20.12.2018

[5] R. Mihalcea and P. Tarau, "TextRank: Bringing Order into Texts", Proceedings of the 2004 Conference on Empirical Methods in Natural Language Processing, (2004), pp. 1-8, available online: http://aclweb.org/anthology/W04-3252, last visit: 21.12.2018

[6] L. Page, S. Brin, R. Motwani and T. Winograd, "The Pagerank Cita-tion Ranking: Bringing Order to the Web", Stanford InfoLab, (1999), pp. 1-17, available online: http://ilpubs.stanford.edu:8090/422/, last visit: 21.12 .2018

[7] F. Barrios, F. López, L. Argerich and R. Wachenchauzer, "Varia-tions of the Similarity Function of TextRank for Automated Sum-marization", Proceedings of Argentine Symposium on Artificial Intelligence (ASAI), (2015), pp. 65-72, available online: http://sedici.unlp.edu.ar/bitstream/handle/10915/52082/Documento_completo.pdfPDFA.pdf?sequence=1\&isAllowed=y, last visit: 21.12 .2018

[8] Y. Wen, H. Yuan and P. Zhang, "Research On Keyword Extraction Based On Word2Vec Weighted Textrank", 2016 2nd IEEE Interna-tional Conference on Computer and Communications (ICCC), (2016), pp. 2109-2113, http://dx.doi.org/10.1109/CompComm.2016.7925072

[9] J. Kupiec, J. Pedersen and F. Chen, "A Trainable Document Sum-marizer", Proceedings of the 18th Annual International ACM SIGIR Conference on Research and Development in Information Retrieval, (1995), pp. 68-73, available http://citeseerx.ist.psu.edu/viewdoc/download?doi=10.1.1.452.7100\&rep=rep1\&type=pdf, last visit: 21.12.2018

[10] D. O'leary and J. Conroy, "Text Summarization Via Hidden Mar-kov Models and Pivoted QR Matrix Decomposition", Proceedings of SIGIR 2001 (the 24th Annual International ACM SIGIR Con-ference on Research and Development in Information Retrieval), (2001), http://dx.doi.org/10.1145/383952.384042

[11] A. Krizhevsky, I. Sutskever and G. E. Hinton, "ImageNet Classifi-cation with Deep Convolutional Neural Networks", Communica-tions of the ACM, Vol. 60, No. 6, (2017), pp. 84-90, http://dx.doi.org/10.1145/3065386

[12] C. N. dos Santos and G. Maira, "Deep Convolutional Neural Net-works for Sentiment Analysis of Short Texts", Proceedings of the 25th International Conference on Computational Linguistics: Technical Papers (COLING 2014), (2014), pp.69-78, available online: http://anthology.aclweb.org/C/C14/C141008.pdf, last vis-it: 21.12 .2018

[13] Y. LeCun, Y. Bengio and G. Hinton, "Deep Learning”, Nature, Vol. 521, (2015), pp. 436-444, https://doi.org/10.1038/nature14539

[14] F. A. Gers, J. Schmidhuber and F. Cummins, "Learning to Forget: Continual Prediction with LSTM", Neural Computation, Vol. 12, No. 10, (2000), pp. 2451-2471, http://dx.doi.org/10.1162/089976600300015015

[15] S. Hochreiter and J. Schmidhuber, "Long Short-Term Memory", Neural Computation, Vol. 9, No. 8, (1997), pp. 1735-1780, http://dx.doi.org/10.1162/neco.1997.9.8.1735 
[16] Y. Wu, M. Schuster, Z. Chen, Q. Le, M. Norouzi, W. Macherey, M. Krikun, Y. Cao, Q. Gao, K. Macherey, J. Klingner, A. Shah, M. Johnson, X. Liu, L. Kaiser, S. Gouws, Y. Kato, T. Kudo, H. Kazawa, K. Stevens, G. Kurian, N. Patil, W. Wang, C. Young, J. Smith, J. Rie-sa, A. Rudnick, O. Vinyals, G. Corrado, M. Hughes and J. Dean, "Google's Neural Machine Translation System: Bridging the Gap Between Human and Machine Translation", arXiv:1609.08144v2, (2016), pp. 1-23.

[17] M. Galeso, "Apple Siri for Mac: An Easy Guide to the Best Fea-tures". First Rank Publishing, (2016)

[18] K. Cho, B. van Merrienboer, D. Bahdanau and Y. Bengio, "On the Properties of Neural Machine Translation: Encoder-Decoder Ap-proaches", Proceedings of SSST-8(Eighth Workshop on Syntax, Semantics and Structure in Statistical Translation), (2014), pp. 103-111, available online: https://www.aclweb.org/anthology/W14-4012, last visit: 21.12.2018

[19] C. Lin, "ROUGE: A Package for Automatic Evaluation of Sum-maries", Proceedings of the PostConference Workshop of ACL 2004 (Text Summarization Branches Out), (2004), pp. 74-82, avail-able online: http://www.aclweb.org/anthology/W04-1013, last visit: 21.12 .2018 
International Journal of Advanced Science and Technology Vol.124 (2019) 